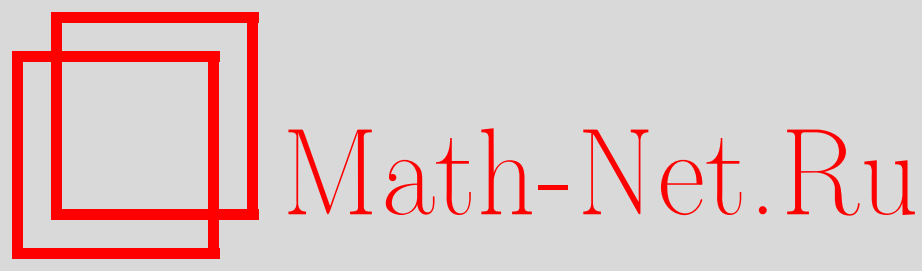

С. Э. Деркачёв, Л. Д. Фаддеев, Замечание о коэффициенте отражения в модели Лиувилля, ТМФ, 2017, том 192, номер 2, 221-226

DOI: https://doi.org/10.4213/tmf9307

Использование Общероссийского математического портала Math-Net.Ru подразумевает, что вы прочитали и согласны с пользовательским соглашением http: //www . mathnet.ru/rus/agreement

Параметры загрузки:

IP : 54.237 .206 .68

26 апреля 2023 г., $17: 36: 18$




Том 192, № 2

август, 2017

(C) 2017 г. $\quad$ С. Э. Деркачев*, Л. Д. Фаддеев *

\section{ЗАМЕЧАНИЕ О КОЭФФИЦИЕНТЕ ОТРАЖЕНИЯ В МОДЕЛИ ЛИУВИЛЛЯ}

Показано, что коэффициенты отражения в квантовой теории модели Лиувилля, вычисленные в рамках бутстрапного и гамильтонова подходов, различаются фазовым множителем и просто дают разные нормировки вертексных операторов.

Ключевые слова: квантовая модель Лиувилля, конформный бутстрап, конформная теория поля.

DOI: https://doi.org/10.4213/tmf9307

\section{1. ВВЕДЕНИЕ}

В квантовой теории модели Лиувилля важную роль играет коэффициент отражения $R(p)$. В бутстрапном подходе [1] он осуществляет эквивалентность вертексных операторов $W_{\alpha}(z)$. В физической области

$$
\alpha=\frac{Q}{2}+i p, \quad p \in \mathbb{R}
$$

где $Q=b+b^{-1}$ и $b$ играет роль константы связи,

$$
W_{\alpha}(z)=R(p) W_{Q-\alpha}(z), \quad|R(p)|=1 .
$$

В операторном подходе $R(p)$ [2]-[5] определяет гильбертово пространство нулевых мод $P, Q$ как подпространство в $L_{2}(\mathbb{R})$, состоящее из функций

$$
\psi(p)=R(p) \psi(-p) .
$$

В работах [4], [5] используются обозначения, принятые в теории автоморфных функций. Если в работе [1] параметр q квантовой группы обозначен как

$$
q=e^{i \pi b^{2}}
$$

Работа выполнена при поддержке Российского научного фонда (проект № 14-11-00598).

* Санкт-Петербургское отделение Математического института им. В. А. Стеклова Российской академии наук, Санкт-Петербург, Россия. E-mail: derkach@pdmi.ras.ru 
то в [4] используется каноническое обозначение

$$
q=e^{i \pi \tau}
$$

с модулярным параметром $\tau$, представленным в виде отношения полупериодов $\omega$ и $\omega^{\prime}$

$$
\tau=\frac{\omega^{\prime}}{\omega}, \quad \omega \omega^{\prime}=-\frac{1}{4} .
$$

Положительному $\tau$ соответствуют $\omega$ и $\omega^{\prime}$ на положительной части мнимой оси, так что

$$
\begin{gathered}
b=\frac{2 \omega^{\prime}}{i}, \quad \frac{1}{b}=\frac{2 \omega}{i}, \\
Q=\frac{2\left(\omega^{\prime}+\omega\right)}{i}=\frac{2 \omega^{\prime \prime}}{i}, \quad \omega^{\prime \prime}=\omega^{\prime}+\omega .
\end{gathered}
$$

Если $\alpha=Q / 2+i p$, то $Q-\alpha=Q / 2-i p$, т. е. замене $\alpha \rightarrow Q-\alpha$ соответствует замена $p \rightarrow-p$. В работах [1] и [4], [5] приведены явные выражения для $R(p)$.

В рамках бутстрапного подхода коэффициент отражения выражается в терминах гамма-функции Эйлера:

$$
\begin{aligned}
R_{Z Z}(p) & =-\mu^{-2 i p} \frac{\Gamma(2 i p b) \Gamma\left(2 i p b^{-1}\right)}{\Gamma(-2 i p b) \Gamma\left(-2 i p b^{-1}\right)}=\frac{Z(-p)}{Z(p)}, \\
Z(p) & =\mu^{i p} \frac{i p}{\pi} \Gamma(-2 i p b) \Gamma\left(-2 i p b^{-1}\right), \quad \overline{Z(p)}=Z(-p),
\end{aligned}
$$

а в рамках операторного подхода в терминах другой специальной функции - квантового дилогарифма:

$$
\begin{aligned}
R_{F V}(p) & =e^{-4 \pi i p \omega^{\prime \prime}} \frac{\gamma\left(-2 p-\omega^{\prime \prime}\right)}{\gamma\left(2 p-\omega^{\prime \prime}\right)}=\frac{M(-p)}{M(p)}, \\
M(p) & =c e^{-2 \pi i p\left(p-\omega^{\prime \prime}\right)} \gamma\left(2 p-\omega^{\prime \prime}\right),
\end{aligned}
$$

где функция $\gamma(x)$ определяется при помощи формулы

$$
\gamma(x)=\exp \left[-\frac{1}{4} \int_{C} \frac{d t}{t} \frac{e^{i t x}}{\sin (t \omega) \sin \left(t \omega^{\prime}\right)}\right] .
$$

Контур $C$ идет вдоль вещественной оси и обходит точку $t=0$ сверху. Константа $c$ выбирается следующим образом:

$$
c=e^{i \beta+i \pi / 4}, \quad \beta=\frac{\pi}{12}\left(\frac{\omega^{\prime}}{\omega}+\frac{\omega}{\omega^{\prime}}\right) .
$$

При таком выборе константы $c$ выполняется соотношение

$$
\overline{M(p)}=M(-p),
$$

которое может быть легко проверено при помощи формул комплексного сопряжения и отражения для квантового дилогарифма

$$
\overline{\gamma(x)}=\frac{1}{\gamma(\bar{x})}, \quad \gamma(x) \gamma(-x)=e^{i \beta} e^{i \pi x^{2}}
$$


и очевидных соотношений

$$
c^{2}=e^{-i \beta} e^{-i \pi \omega^{\prime \prime 2}}, \quad \bar{c}=c^{-1} .
$$

На первый взгляд, формулы (4) и (6) совсем не похожи, и несколько лет оставалась неясной причина такого расхождения бутстрапного и гамильтонова подходов. Мы покажем, что на самом деле $R_{Z Z}(p)$ и $R_{F V}(p)$ различаются фазовым множителем и просто дают разные нормировки операторов $W_{\alpha}(z)$.

\section{2. ПЕРЕНОРМИРОВКА}

Если изменить нормировку $W_{\alpha}^{\prime}(z)=\lambda(p) W_{\alpha}(z)$, то для перенормированного вертексного оператора получим

$$
\begin{aligned}
W_{\alpha}^{\prime}(z) & =R^{\prime}(p) W_{Q-\alpha}^{\prime}(z), \\
R^{\prime}(p) & =\frac{\lambda(p)}{\lambda(-p)} R(p),
\end{aligned}
$$

т. е. коэффициент отражения при перенормировке изменяется по простому закону. Мы хотим найти функцию $\lambda(p)$, связывающую коэффициенты отражения в двух подходах:

$$
R_{F V}(p)=\frac{\lambda(p)}{\lambda(-p)} R_{Z Z}(p)
$$

или, явным образом,

$$
\frac{\lambda(p)}{\lambda(-p)}=\frac{M(-p) Z(p)}{M(p) Z(-p)} .
$$

Самое простое решение имеет вид

$$
\lambda(p)=\frac{M(-p)}{Z(-p)}, \quad \overline{\lambda(p)}=\lambda(-p),
$$

и удовлетворяет дополнительному ограничению

$$
\overline{\lambda(p)} \lambda(p)=\lambda(p) \lambda(-p)=\frac{M(-p) M(p)}{Z(-p) Z(p)}=1,
$$

справедливость которого следует из тождества

$$
Z(p) Z(-p)=M(p) M(-p)=-\frac{1}{4 \sin (2 \pi i p b) \sin \left(2 \pi i p b^{-1}\right)} .
$$

Вычисление первого произведения сводится к использованию соотношения отражения для гамма-функции Эйлера

$$
\Gamma(z) \Gamma(-z)=-\frac{\pi}{z \sin (\pi z)},
$$

так что получаем

$$
\begin{aligned}
Z(p) Z(-p) & =\frac{p^{2}}{\pi^{2}} \Gamma(-2 i p b) \Gamma(2 i p b) \Gamma\left(-2 i p b^{-1}\right) \Gamma\left(2 i p b^{-1}\right)= \\
& =-\frac{1}{4 \sin (2 \pi i p b) \sin \left(2 \pi i p b^{-1}\right)} .
\end{aligned}
$$


При вычислении второго произведения используется соотношение

$$
\frac{\gamma\left(x+\omega^{\prime \prime}\right)}{\gamma\left(x-\omega^{\prime \prime}\right)}=-4 e^{2 \pi i x \omega^{\prime \prime}} \sin \left(\frac{\pi x}{2 \omega}\right) \sin \left(\frac{\pi x}{2 \omega^{\prime}}\right)
$$

которое легко выводится из определяющих функциональных уравнений для квантового дилогарифма

$$
\frac{\gamma\left(x+\omega^{\prime}\right)}{\gamma\left(x-\omega^{\prime}\right)}=1+e^{-(i \pi / \omega) x}, \quad \frac{\gamma(x+\omega)}{\gamma(x-\omega)}=1+e^{-\left(i \pi / \omega^{\prime}\right) x} .
$$

При помощи формулы отражения (8) и соотношений (12), (9) получаем

$$
\begin{aligned}
M(p) M(-p) & =c^{2} e^{-4 \pi i p^{2}} \gamma\left(2 p-\omega^{\prime \prime}\right) \gamma\left(-2 p-\omega^{\prime \prime}\right)= \\
& =c^{2} e^{-4 \pi i p^{2}} e^{i \beta} e^{i \pi\left(2 p+\omega^{\prime \prime}\right)^{2}} \frac{\gamma\left(2 p-\omega^{\prime \prime}\right)}{\gamma\left(2 p+\omega^{\prime \prime}\right)}=-\frac{1}{4 \sin (\pi p / \omega) \sin \left(\pi p / \omega^{\prime}\right)} .
\end{aligned}
$$

Таким образом, коэффициенты отражения в двух подходах связаны соотношением

$$
R_{F V}(p)=\lambda^{2}(p) R_{Z Z}(p)
$$

где

$$
\lambda(p)=\frac{M(-p)}{Z(-p)}, \quad|\lambda(p)|^{2}=\lambda(p) \lambda(-p)=1,
$$

т. е. просто различаются фазовым множителем.

2.1. Коэффициент отражения и двухточечная функция Грина. Рассмотрим связь коэффициента отражения с двухточечной функцией Грина. С точностью до общей нормировки $N(p)$ двухточечная функция Грина имеет вид

$$
\left\langle W_{\alpha}\left(z_{1}\right) W_{\alpha^{\prime}}\left(z_{2}\right)\right\rangle=\frac{N(p)}{\left|z_{1}-z_{2}\right|^{2 \Delta(p)}}\left(\delta\left(p+p^{\prime}\right)+R(p) \delta\left(p-p^{\prime}\right)\right),
$$

где $\Delta(p)$ - конформная размерность оператора $W_{\alpha}(z)$,

$$
\Delta(p)=\alpha(Q-\alpha)=\frac{Q^{2}}{4}+p^{2}
$$

Формула (16) диктуется глобальной конформной инвариантностью. Глобальные конформные преобразования - это преобразования из группы Мёбиуса или группы дробно-линейных преобразований. Зависимость от $z_{1}$ и $z_{2}$ однозначно определяется требованиями трансляционной и масштабной инвариантности, а инвариантность относительно инверсий приводит к тому, что рассматриваемая функция Грина отлична от нуля только при совпадении конформных размерностей полей $\Delta(p)=\Delta\left(p^{\prime}\right)$. Явное представление для коэффициента в формуле $(16)$ - следствие соотношения (10) и дополнительного требования, чтобы коэффициент при $\delta\left(p+p^{\prime}\right)$ был равен единице. 
Если изменить нормировку $W_{\alpha}^{\prime}=\lambda(p) W_{\alpha}$, то для функций Грина новых полей получим

$$
\left\langle W_{\alpha}^{\prime}\left(z_{1}\right) W_{\alpha^{\prime}}^{\prime}\left(z_{2}\right)\right\rangle=\frac{N(p)}{\left|z_{1}-z_{2}\right|^{2 \Delta(p)}}\left(\lambda(p) \lambda(-p) \delta\left(p+p^{\prime}\right)+R(p) \lambda^{2}(p) \delta\left(p-p^{\prime}\right)\right) .
$$

Таким образом, преобразование перенормировки, которое связывает коэффициенты отражения $R_{Z Z}(p)$ и $R_{F V}(p)$,

$$
R_{F V}(p)=\lambda^{2}(p) R_{Z Z}(p), \quad|\lambda(p)|^{2}=\lambda(p) \lambda(-p)=1,
$$

не меняет общее выражение для двухточечной функции Грина (16), преобразуя лишь коэффициент отражения $R(p)$ из $R_{Z Z}(p)$ в $R_{F V}(p)$.

В заключение отметим, что коэффициенты отражения в двух подходах могут быть выражены при помощи гамма-функции Барнса

$$
\Gamma_{b}(x)=\exp \left\{\int_{0}^{\infty} \frac{d t}{t}\left[\frac{e^{-t x}-e^{-t Q / 2}}{\left(1-e^{-t b}\right)\left(1-e^{-t b^{-1}}\right)}-\frac{1}{2}\left(\frac{Q}{2}-x\right)^{2} e^{-t}-\left(\frac{Q}{2}-x\right) \frac{1}{t}\right]\right\} .
$$

Функция $\Gamma_{b}(x)$ является решением системы функциональных уравнений

$$
\frac{\Gamma_{b}(x+b)}{\Gamma_{b}(x)}=\frac{(2 \pi)^{1 / 2} b^{x b-1 / 2}}{\Gamma(x b)}, \quad \frac{\Gamma_{b}\left(x+b^{-1}\right)}{\Gamma_{b}(x)}=\frac{(2 \pi)^{1 / 2} b^{-x b^{-1}+1 / 2}}{\Gamma\left(x b^{-1}\right)},
$$

где $\Gamma(x)$ - гамма-функция Эйлера. При помощи формулы

$$
\frac{\Gamma_{b}(z+Q)}{\Gamma_{b}(z)}=\frac{2 \pi}{z} \frac{b^{z\left(b-b^{-1}\right)}}{\Gamma\left(z b^{-1}\right) \Gamma(z b)}
$$

являющейся непосредственным следствием соотношений (19), легко вывести следующее представление для коэффициента отражения (4) (с учетом (1)):

$$
R_{Z Z}(\alpha)=\left[\mu b^{-2\left(b-b^{-1}\right)}\right]^{Q-2 \alpha} \frac{\Gamma_{b}(2 \alpha-Q) \Gamma_{b}(2 Q-2 \alpha)}{\Gamma_{b}(2 \alpha) \Gamma_{b}(Q-2 \alpha)} .
$$

Квантовый дилогарифм можно представить в виде отношения двух функций Барнса,

$$
\gamma(x)=\frac{\Gamma_{b}(Q / 2-i x)}{\Gamma_{b}(Q / 2+i x)}, \quad \gamma\left(2 p-\omega^{\prime \prime}\right)=\frac{\Gamma_{b}(Q-2 \alpha)}{\Gamma_{b}(2 \alpha)}
$$

так что для коэффициента отражения (6) получаем

$$
R_{F V}(\alpha)=e^{\pi i(Q-2 \alpha)^{2}} \frac{\Gamma_{b}(2 \alpha) \Gamma_{b}(2 \alpha-Q)}{\Gamma_{b}(Q-2 \alpha) \Gamma_{b}(2 Q-2 \alpha)} .
$$

Выражения (21) и (22) сразу подсказывают выбор нормировки:

$$
R_{F V}(\alpha)=\lambda^{2}(\alpha) R_{Z Z}(\alpha), \quad \lambda(\alpha)=\left[e^{\pi i(2 \alpha-Q)} \mu b^{-2\left(b-b^{-1}\right)}\right]^{\alpha-Q / 2} \frac{\Gamma_{b}(2 \alpha)}{\Gamma_{b}(2 Q-2 \alpha)} .
$$




\section{Список литературы}

[1] A B. Zamolodchikov, Al. B. Zamolodchikov, "Conformal bootstrap in Liouville field theory", Nucl. Phys. B, 477:2 (1996), 577-605; Structure constants and conformal bootstrap in Liouville field theory, arXiv: hep-th/9506136.

[2] L. D. Faddeev, L. A. Takhtajan, "Liouville model on the lattice", Field Theory, Quantum Gravity and Strings (Observatoire de Meudon, and LPTHE, Université Pierre et Marie Curie, Paris, 1984-1985), Lecture Notes in Physics, 246, eds. H. J. de Vega, N. Sanchez, Springer, Berlin, 1986, 166-179.

[3] J. Teschner, "Liouville theory revisited", Class. Quantum Grav., 18:23 (2001), R153-R222, arXiv: hep-th/0104158.

[4] L. D. Faddeev, A. Yu. Volkov, "Discrete evolution for the zero-modes of the quantum Liouville model", J. Phys. A, 41:19 (2008), 194008, 12 pp., arXiv: 0803.0230.

[5] Л. Д. Фаддеев, "Нулевые моды для квантовой модели Лиувилля", Функи. анализ и его прил., 48:3 (2014), 14-23, arXiv: 1404.1713. 